\title{
Adjustable Bandwidth Filter Design Based on Interdigital Capacitors
}

\author{
Li Zhu, Vijay Devabhaktuni, Member, IEEE, Chunyan Wang, Senior Member, IEEE, and \\ Ming Yu, Senior Member, IEEE
}

\begin{abstract}
In this letter, we explore a general-purpose microstrip coupling model for computer aided design of new bandpass filters. The J-inverter topology of the model facilitates the study of coupling behaviours of different microstrip structures leading to a quick comparison of their coupling strengths. Based on such comparison, microstrip filters with interdigital capacitors and etched slots yielding relatively higher coupling coefficients are designed, fabricated and tested. The filters exhibit relatively wider bandwidths which are easily adjustable by way of changing the geometrical parameters of interdigital capacitors and slots.
\end{abstract}

Index Terms-Bandpass filters (BPFs), electromagnetic (EM) coupling, interdigital capacitors.

\section{INTRODUCTION}

$\mathbf{M}$ ICROWAVE filters are essential components of modern communication systems [1]. Microstrip hairpin resonators are widely used in multistage bandpass filters (BPFs) [2]. In contrast to traditional cross-coupled filters [3], hairpin-resonator filters offer two transmission zeros lying on either sides of the passband, sharper cut-off frequencies and lower insertion losses. The design of hairpin-resonator filters with wider bandwidths involves reducing gap and strip widths of resonators in order to achieve stronger electromagnetic (EM) coupling. However, such an approach leads to a degradation of the filtering behavior, i.e., a lower $Q$ and higher loss, and could result in difficulties in the fabrication process, i.e., requirement for increased precision with respect to gap/strip widths and conductor thickness. In this letter, we explore a $J$-inverter topology [4], [5] to estimate EM coupling strengths of different coupling structures. Based on the study, compact filters using interdigital capacitors and etched slots, with relatively stronger coupling are designed, fabricated and tested. It is shown that the bandwidths of these filters can be easily adjusted by changing the geometrical parameters of the interdigital capacitors and/or the etched slots.

Manuscript received July 10, 2007; revised September 12, 2007. This work was supported by the Natural Sciences and Engineering Research Council (NSERC) of Canada and the Canada Research Chairs (CRC) program.

L. Zhu, V. Devabhaktuni, and C. Wang are with the Department of Electrical and Computer Engineering, Concordia University, Montreal, QC H3G 1M8 Canada (e-mail: vijayd@ece.concordia.ca).

M. Yu is with COM DEV, Ltd., Cambridge, ON N1R 7H6 Canada (e-mail: ming.yu@ieee.org).

Color versions of one or more of the figures in this letter are available online at http://ieeexplore.ieee.org.

Digital Object Identifier 10.1109/LMWC.2007.911975

\section{ESTIMATION OF EM COUPLING}

Different microstrip coupling structures, illustrated in Fig. 1, are considered. Fig. 1(a) shows an unbound end-to-end coupling gap with open-ends $R_{1}$ and $R_{2}$, and Fig. 1(b) shows a nine-finger interdigital capacitor. The structure in Fig. 1(c) is identical to that in Fig. 1(b) except that it has a rectangular ground-plane aperture below the interdigital capacitor. Based on a short-open calibration scheme [4], two-port coupled microstrip lines can be characterized by a $J$-inverter network model [5]. In Fig. 1(d), an equivalent $J$-inverter network model for the coupling structures is presented. The physical structure is modeled as an equivalent $J$-inverter network combined with two identical error terms $\left[X_{f}\right]$. The error terms relate to the approximation of source excitation and inconsistency between 2-D and 3-D method of moments (MOM)-based impedance definitions [4].

Specifically, the $J$-inverter network consists of susceptance $(J)$ and two equivalent electrical line lengths $(\theta / 2$ each). For a symmetrical two-port $J$-inverter network [5], we have

$$
\frac{J}{Y_{0}}=\frac{\tan (\theta / 2)+\bar{B}_{11}}{\bar{B}_{12} \tan (\theta / 2)}
$$

and

$$
\theta=-\tan ^{-1}\left(\frac{2 \bar{B}_{11}}{1-\bar{B}_{11}^{2}+\bar{B}_{12}^{2}}\right)
$$

where $Y_{0}$ is the characteristic admittance of each line, $B_{i j}$ is the susceptance of the corresponding $Y$-parameter from simulation, and $\bar{B}_{i j}=B_{i j} / Y_{0}$. Equations (1) and (2) allow transformation from an admittance $\pi$-network to a $J$-inverter topology [5]. Fig. 2 shows normalized values of $J$ for different coupling structures in the $1-8 \mathrm{GHz}$ range. Clearly, $J$ varies nonlinearly with frequency $f$, exhibiting frequency dispersion behavior. $J$ increases noticeably from the structure of Fig. 1(a) to the structures of Fig. 1(b) and (c), with the structure of Fig. 1(c) exhibiting largest $J$. Since $J$ is directly proportional to coupling strength [6], it can be inferred that coupling strength can be enhanced via use of interdigital capacitors and/or etched slots.

\section{PROPOSED BPF STRCUTURES}

A larger $J$ implies a stronger coupling or smaller external quality-factor $\left(Q_{E}\right)$ of a resonator [6]. Motivated by this, we propose new BPFs based on the coupling structures of Section II. The $3 \mathrm{~dB}$ bandwidth of a filter can be expressed as

$$
\Delta f_{3 \mathrm{~dB}}=\frac{f_{0}}{\left(Q_{E} / 2\right)}
$$




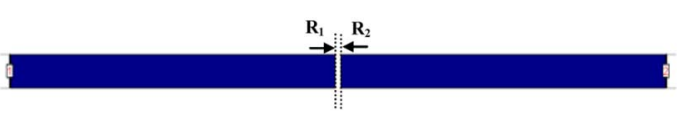

(a)

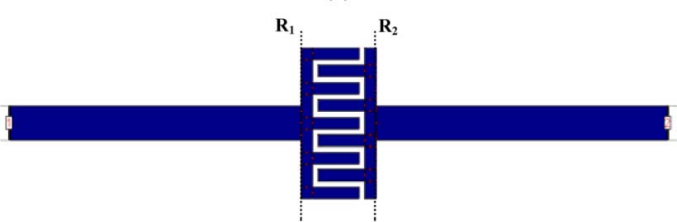

(b)

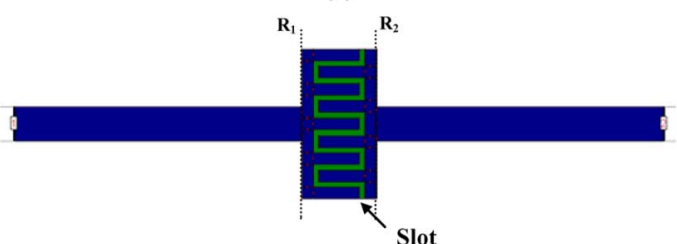

(c)

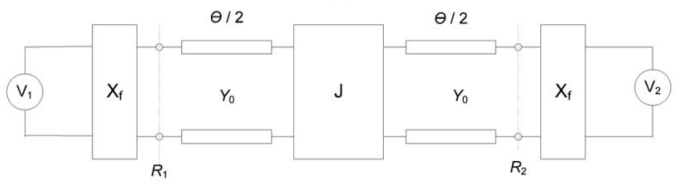

(d)

Fig. 1. (a) End-to-end coupled line, (b) interdigital capacitor coupled line, (c) interdigital capacitor coupled line with etched slot, and (d) generalized $J$-inverter network model for the coupling structures.

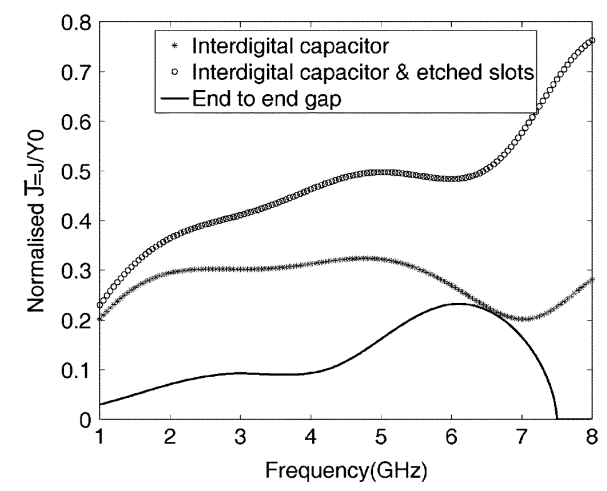

Fig. 2. Normalized susceptance $\left(J / Y_{0}\right)$ for the coupling structures of Fig. 1.

where $f_{0}$ denotes centre frequency. A larger $J$ results in a smaller $Q_{E}$ and hence a wider bandwidth. Different bandwidths can be achieved by choosing coupling structures with different $J$. In the following sub-section, the design of two kinds of new BPFs is proposed.

\section{A. Proposed BPF}

Interdigital capacitors are used to substitute for the traditional gaps between hairpin resonators, thereby facilitating stronger coupling. As shown in Fig. 3(b), two interdigital capacitors, each with 4 fingers, are used. In this structure, the width of the microstrip hairpin is $1 \mathrm{~mm}$. The length and width of the fingers are $0.3 \mathrm{~mm}$ and $0.2 \mathrm{~mm}$. Each gap between fingers is $0.2 \mathrm{~mm}$ where that of the traditional structure [see Fig. 3(a)] is set to 0.13 $\mathrm{mm}$ (or $5 \mathrm{mil}$ ), which is the closest/smallest distance allowed by the fabrication process employed. Substrate thickness $(h)$ is

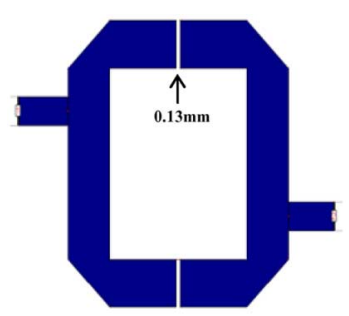

(a)

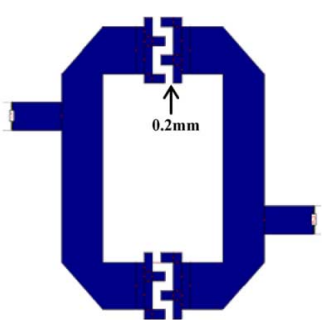

(b)

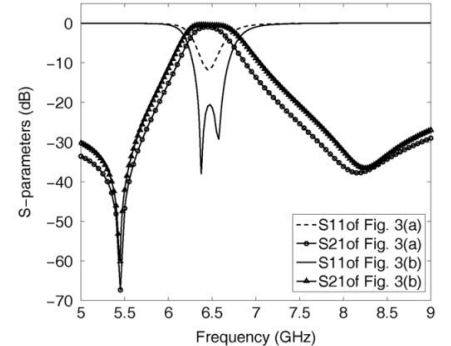

(c)

Fig. 3. (a) Layout of a traditional edge-coupled BPF, (b) layout of the proposed BPF 1, and (c) comparison of simulated $S$-parameters of the proposed filter 1 and the traditional filter.

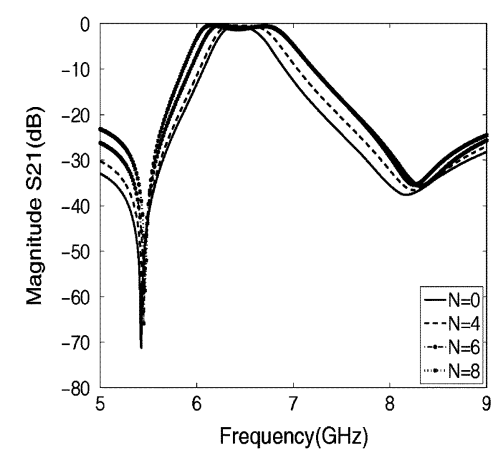

Fig. 4. $S_{21}$ of the proposed BPFs 1 with different values of $N$.

$0.635 \mathrm{~mm}$ and dielectric constant $\left(\varepsilon_{r}\right)$ is 10.2 . The results based on EM simulations of Zeland IE3D, shown in Fig. 3(c), compare the bandpass behaviors of the proposed and the traditional filters. Owing to a stronger coupling effect, the proposed filter exhibits a wider $3 \mathrm{~dB}$ bandwidth (602 MHz versus $375 \mathrm{MHz}$ ), while its $f_{0}$ remains unchanged at $6.5 \mathrm{GHz}$. For the proposed filter, an insertion loss of $0.41 \mathrm{~dB}$ at $6.49 \mathrm{GHz}$ and a return loss $>20 \mathrm{~dB}$ in the $6.34-6.61 \mathrm{GHz}$ range are observed.

It is possible to further widen the bandwidth of the proposed filter by increasing the number of fingers $(N)$, while keeping $f_{0}$ fixed by slightly adjusting the lengths of the hairpin resonators. $I E 3 D$ simulations of the proposed filter with different values of $N$ are performed and the results are illustrated in Fig. 4. A summary of the simulation results is presented in Table I. Bandwidth increases with $N$, while $f_{0}$ and locations of transmission zeros remain more or less unchanged. Changing $N$ from 0 (i.e., traditional gap) to 8 leads to an increase in $3 \mathrm{~dB}$ fractional bandwidth (FBW) from $5.8 \%$ to $15.5 \%$, and a change in $Q_{E}$ from 34.7 to 12.9 , thus indicating a considerable degree of design flexibility. However, it may be difficult to design a filter with FBW $>20 \%$, using the proposed structure, since all line resonators are highly dependent on frequency [7]. 
TABLE I

SUMMARY OF IE3D SIMULATIONS FOR DIFFERENT VALUES OF $N$

\begin{tabular}{|c|c|c|}
\hline $\begin{array}{c}\text { No. of } \\
\text { Fingers }\end{array}$ & $\begin{array}{c}\text { 3dB } \\
\text { Bandwidth / } \\
\boldsymbol{F} \boldsymbol{B} \boldsymbol{W}\end{array}$ & $\boldsymbol{Q}_{\mathbf{E}}$ \\
\hline 0 & $\begin{array}{c}375 \mathrm{MHz} / \\
5.8 \%\end{array}$ & 34.7 \\
\hline 4 & $\begin{array}{c}602 \mathrm{MHz} / \\
9.3 \%\end{array}$ & 21.6 \\
\hline 6 & $\begin{array}{c}841 \mathrm{MHz} / \\
12.9 \%\end{array}$ & 15.5 \\
\hline 8 & $\begin{array}{c}1005 \mathrm{MHz} / \\
15.5 \%\end{array}$ & 12.9 \\
\hline
\end{tabular}

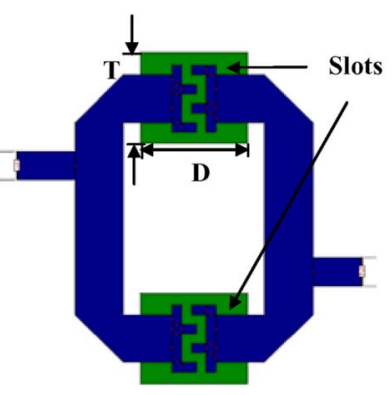

(a)

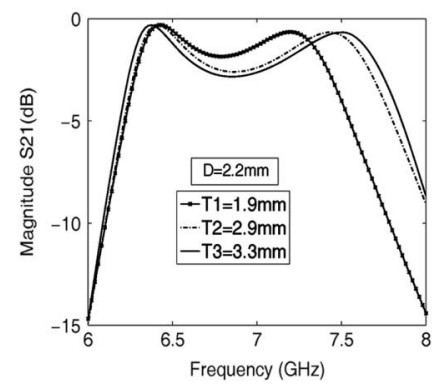

(b)
Fig. 5. (a) Layout of the proposed filter with etched slots and (b) its simulated $S_{21}$ for different values of $T$.

\section{B. Proposed BPF 2}

To further improve the bandwidth, two rectangular slots are etched in the ground plane right below the four-finger interdigital capacitors as shown in Fig. 5(a). As can be seen in Fig. 5(b), increasing the width $(T)$ of the etched slots from 1.9 to $3.3 \mathrm{~mm}$ while keeping other geometrical parameters unchanged, results in an increase in $3 \mathrm{~dB}$ FBW from $17.4 \%$ to $22.7 \%$ and a change in $Q_{E}$ from 11.5 to 8.8. It is to be noted that the simultaneous change (i.e., increase) in $f_{0}$ can be countered by slightly increasing $N$ alone. The proposed filters with etched slots offer a significant flexibility in terms of broadband filter design.

\section{FABRICATION AND MEASUREMENT}

The proposed filters are fabricated on a process of $0.635 \mathrm{~mm}$ thick RT/duroid 6010.2 substrate with $\varepsilon_{r}=10.2$. The photograph and $S$-parameters of the eight-finger BPF 1 are shown in Fig. 6. Measurements are performed using an Agilent 8510C VNA. The filter has $f_{0}=6.5 \mathrm{GHz}$ and $3 \mathrm{~dB} \mathrm{FBW}=15.6 \%$, which agree with $I E 3 D$ simulations of Table I. A return loss $>15 \mathrm{~dB}$ in the 6.02-6.44 GHz passband, and an insertion loss $>1 \mathrm{~dB}$ in the $6.01-6.41 \mathrm{GHz}$ range are observed. Fig. 7 shows a photograph and the measured/simulated $S$-parameters of the fabricated four-finger BPF 2 with etched slots. The filter has $f_{0}=7 \mathrm{GHz}$ and $3 \mathrm{~dB} \mathrm{FBW}=23.1 \%$. A return loss $>15 \mathrm{~dB}$ in the 6.62-7.24 GHz passband and an insertion loss $>1 \mathrm{~dB}$ in the 6.50-7.26 GHz range are observed. The discrepancies between measurements and simulations can be attributed to fabrication tolerances (considering high sensitivity of the ring resonators w.r.t. $\varepsilon_{r}$ and $h$ [8]) and to some extent to calibration errors.

\section{CONCLUSION}

A general-purpose circuit model has been studied and applied to the estimation of EM couplings of different coupling struc-

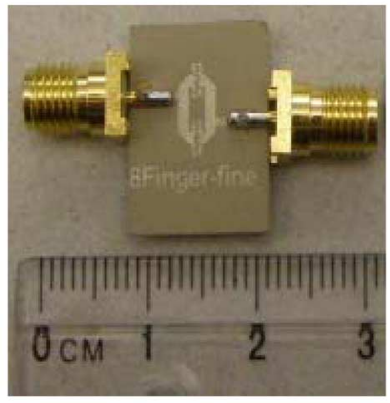

(a)

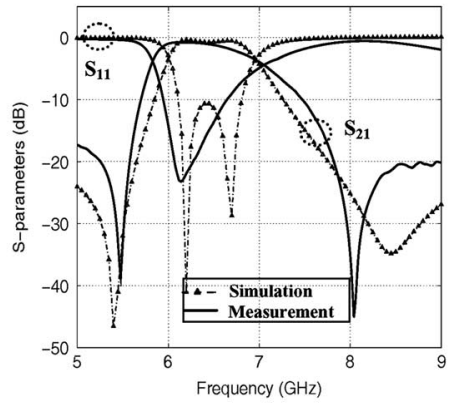

(b)
Fig. 6. (a) Photograph of an eight-finger BPF 1 and (b) its measured and simulated $S$-parameters.

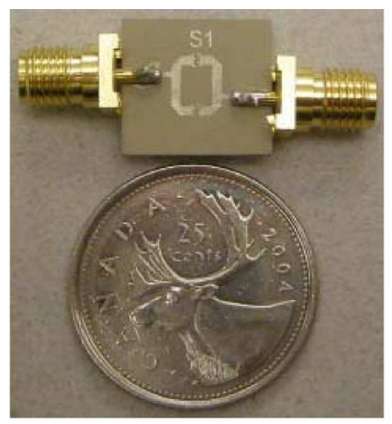

(a)

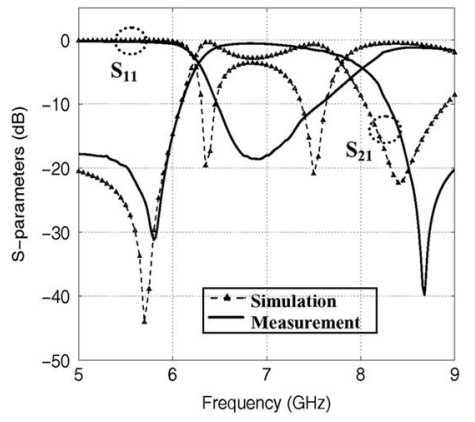

(b)
Fig. 7. (a) Photograph of a four-finger BPF 2 with etched slots and (b) its measured and simulated $S$-parameters. For this filter, $T=3.3 \mathrm{~mm}$.

tures. Based on the study, two new microstrip filters employing interdigital capacitors and etched slots have been designed, fabricated, and tested. Both experiment results and EM simulations have shown that the proposed filters offer wider bandwidths compared to edge-coupled filters. The Bandwidths of the new filters can be adjusted by changing their geometrical parameters. It has also been shown that the proposed filers offer a considerable degree of design flexibility.

\section{REFERENCES}

[1] G. L. Matthaei, L. Young, and E. M. T. Jones, Microwave Filters, Impedance-Matching Networks, and Coupling Structures. Norwood, MA: Artech House, 1980.

[2] L. H. Hsieh and K. Chang, "Tunable microstrip bandpass filters with two transmission zeros," IEEE Trans. Microw. Theory Tech., vol. 51, no. 2, pp. 520-525, Feb. 2003.

[3] S. Y. Lee and C. M. Tsai, "New cross-coupled filter design using improved hairpin resonators," IEEE Tran. Microw. Theory Tech., vol. 48, no. 12, pp. 2482-2490, Dec. 2000.

[4] L. Zhu and K. Wu, "Unified equivalent-circuit model of planar discontinuities suitable for field theory-based CAD and optimization of M(H)MIC," IEEE Trans. Microw. Theory Tech., vol. 45, no. 9, pp. 2114-2124, Sep. 1999.

[5] L. Zhu and $\mathrm{K}$. Wu, "Accurate circuit model of interdigital capacitor and its application to design of new quasi-lumped miniaturized filters with suppression of harmonic resonance," IEEE Trans. Microw. Theory Tech., vol. 48, no. 3, pp. 347-356, Mar. 2000.

[6] J. S. Hong and M. J. Lancaster, Microstrip Filters for RF/Microwave Applications. New York: Wiley, 2001.

[7] L. Zhu, H. Bu, and K. Wu, "Broadband and compact multipole microstrip bandpass filters using ground plane aperture technique," Proc. Inst. Elect. Eng., vol. 149, pp. 71-77, 2002.

[8] K. Chang and L. H. Hsieh, Microwave Ring Circuits and Related Structures. New York: Wiley, 2004. 